Akadémiai Kiadó - Springer-Verlag

\title{
TURÁN-RAMSEY THEOREMS \\ AND SIMPLE ASYMPTOTICALLY EXTREMAL STRUCTURES
}

\author{
P. ERDÖS, A. HAJNAL, M. SIMONOVITS, V. T. SÓS and E. SZEMERÉDI
}

Received October 31, 1989

This paper is a continuation of [10], where P. Erdös, A. Hajnal, V. T. Sós, and E. Szemerédi investigated the following problem:

Assume that a so called forbidden graph $L$ and a function $f(n)=o(n)$ are fixed. What is the maximum number of edges a graph $G_{n}$ on $n$ vertices can have without containing $L$ as a subgraph, and also without having more than $f(n)$ independent vertices?

This problem is motivated by the classical Turán and Ramsey theorems, and also by some applications of the Turán theorem to geometry, analysis (in particular, potential theory) [27-29], [11-13].

In this paper we are primarily interested in the following problem. Let $\left(G_{n}\right)$ be a graph sequence where $G_{n}$ has $n$ vertices and the edges of $G_{n}$ are coloured by the colours $\chi_{1}, \ldots, \chi_{r}$, so that the subgraph of colour $\chi_{\nu}$ contains no complete subgraph $K_{p_{\nu}},(\nu=1, \ldots, r)$. Further, assume that the size of any independent set in $G_{n}$ is $o(n)($ as $n \rightarrow \infty)$. What is the maximum number of edges in $G_{n}$ under these conditions?

One of the main results of this paper is the description of a procedure yielding relatively simple sequences of asymptotically extremal graphs for the problem. In a continuation of this paper we shall investigate the problem where instead of $\alpha\left(G_{n}\right)=o(n)$ we assume the stronger condition that the maximum size of a $K_{p}$-free induced subgraph of $G_{n}$ is $o(n)$.

Notation. In this paper we shall primarily consider graphs without loops and multiple edges. However, (as tools to prove our results) we shall also use coloured graphs with weighted edges and vertices. Given a graph $G, e(G)$ will denote the number of its edges, $v(G)$ the number of its vertices, $\chi(G)$ its chromatic number, $\alpha(G)$ the maximum size of an independent set in it. Given a graph, the (first) subscript will denote the number of vertices: $G_{n}, S_{n}, \ldots$ will always denote graphs on $n$ vertices. $R\left(k_{1}, \ldots, k_{r}\right)$ will denote the usual Ramsey number, i.e., the minimum $t$ such that for every edge colouring of $K_{t}$ in $r$ colours $K_{t}$ contains a monochromatic $K_{k_{\nu}}$ for some colour $\chi_{\nu}$. One more convention on the colouring of graphs: whenever we use two colours $\chi_{1}$ and $\chi_{2}$, we shall call the first colour RED, the second one BLUE.

Given two disjoint vertex sets, $X$ and $Y$, in a graph $G_{n}, e_{G}(X, Y)$ denotes the number of edges joining $X$ and $Y$, and $d_{G}(X, Y)$ denotes the edge-density between them:

$$
d_{G}(X, Y)=\frac{e_{G}(X, Y)}{|X| \cdot|Y|}
$$


The number of edges in a subgraph spanned by a set $X$ of vertices of $G$ will be denoted by $e_{G}(X)$. We shall say that $X$ is "completely joined" to $Y$ if every vertex of $X$ is joined to every vertex of $Y$.

Given two points $x, y$ in the Euclidean space $\mathbf{E}^{h}, \rho(x, y)$ will denote their distance.

Given two graphs $G_{n}$ and $H_{n}$, their distance $\Delta\left(G_{n}, H_{n}\right)$ is defined as the minimum number of edges one has to delete from and add to $G_{n}$ to get a graph isomorphic to $H_{n}$.

\section{Introduction}

This paper is a continuation of [10], where P. Erdős, A. Hajnal, V. T. Sós, and E. Szemerédi, investigated the following problem:

Assume that a so called forbidden graph $L$ and a function $f(n)=o(n)$ are given. What is the maximum number of edges a graph $G_{n}$ can have without containing $L$ as a subgraph, and also without having more than $f(n)$ independent vertices?

This problem is motivated by the classical Turán and Ramsey theorems $[25,26]$, [19], (see also [1,21]), and also by some applications of the Turán theorem to geometry, analysis (in particular, potential theory) [27-29], [11-13].

In 1930 Ramsey proved his famous theorem [19]:

Ramsey theorem for ordinary graphs. Given $r$ integers $k_{1}, \ldots, k_{r}$, there exists a threshold integer $R=R\left(k_{1}, \ldots, k_{r}\right)$ such that if a complete graph $K_{n}$ is edgecoloured in $r$ colours and $n \geq R$, then for some $\nu \leq r$ it contains a $K_{k_{\nu}}$ in the $\nu$ th colour.

Motivated by this theorem, Turán posed the following question:

What is the maximum number of edges a graph $G_{n}$ can have without containing a complete $K_{q}$ ?

Obviously, if we partition $n$ vertices into $q-1$ classes as equally as possible and join two vertices iff they belong to different classes, then we obtain a graph not containing $K_{q}$. This graph will be denoted by $T_{n, q-1}$, and called the Turán graph on $n$ vertices and $q-1$ classes.

P. Turán proved (1940) $[25,26]$, that

Turán theorem. Given $n$ and $q,(1<q \leq n)$, all the graphs $G_{n}$ on $n$ vertices not containing a $K_{q}$ have at most $t(n, q-1)=e\left(T_{n, q-1}\right)$ edges, and this maximum is attained only by $T_{n, q-1}$.

Note that

$$
t(n, q-1)=\left(1-\frac{1}{q-1}\right)\left(\begin{array}{l}
n \\
2
\end{array}\right)+O(1) .
$$

As Turán observed, both Ramsey's and his theorems are, in some sense, generalizations of the Pigeon Hole Principle, and therefore they are applicable in many different situation [21]. He himself started a new line of applications [27-29], [1113 in geometry and analysis (primarily potential theory). Another line was started by G. Katona, where some Turán type theorems were used to obtain inequalities in Probability Theory. One of the limitations of these applications seemed to be that 
in Turán's theorem the graphs attaining the maximum (called extremal graphs) are too special e.g. they have "large" independent sets. The question is, how stable the extremal graph is? In [22] the following was asked:

How many edges can a graph $G_{n}$ have if it contains neither a $K_{q}$ nor such an "enormous" independent set?

Since that a whole theory has emerged around this and similar questions. We first formulate a general question in this field.

Question. Let $L_{1}, \ldots, L_{r}$ be given graphs and a graph $G_{n}$ on $n$ vertices be coloured by $r$ colours $\chi_{1}, \ldots, \chi_{r}$. Assume that the subgraph of colour $\chi_{\nu}$ contains no $L_{\nu}$, (for $\nu=1, \ldots, r$ ), and $\alpha\left(G_{n}\right) \leq m$. What is the maximum of $e\left(G_{n}\right)$ under these conditions? This maximum will be denoted by $R T\left(n, L_{1}, \ldots, L_{r}, m\right)$, and the $L_{\nu}$ 's will be called forbidden graphs.

In the case when the forbidden graphs are complete graphs, $L_{\nu}=K_{k_{\nu}}$, we shall use the simpler notation $R T\left(n, k_{1}, \ldots, k_{r}, m\right)$. Mainly we will be interested in the case $m=f(n)=o(n)$, and use the (simplified) notation $R T\left(n, k_{1}, \ldots, k_{r}, o(n)\right)$.

In [10] primarily the problem of $R T(n, L, o(n))$, i.e. the case of $r=1$ and arbitrary $L$ was investigated. Here we consider the problem for $r>1$.

It is probably hopeless to give an exact description of the optimum. Quite often, instead of looking for the optimal (or so-called extremal) graphs, we try to find an asymptotically extremal sequence of graphs of relatively simple structure. "Relatively simple" means that its structure depends very loosely on $n$.

Definition 1. (Asymptotically extremal graphs) Given the forbidden graphs $L_{1}, \ldots, L_{r}$, and the function $f$, a sequence of graphs $\left(S_{n}\right)$ will be called an asymptotically extremal sequence for $R T\left(n, L_{1}, \ldots, L_{r}, f(n)\right)$ if the edges of $S_{n}$ can be $r$-coloured so that the $\nu$ th colour contains no $L_{\nu}$, for $\nu=1, \ldots, r, \alpha\left(S_{n}\right) \leq f(n)$ and. $e\left(S_{n}\right)=R T\left(n, L_{1}, \ldots, L_{r}, f(n)\right)+o\left(n^{2}\right)$.

In this paper we give upper bounds on $R T\left(n, L_{1}, \ldots, L_{r}, o(n)\right)$ and show that a generalization of the Bollobás-Erdős graph [2] forms an asymptotically extremal sequence for the problem of $R T\left(n, k_{1}, \ldots, k_{r}, o(n)\right)$. In Sections 2,3 we shall define the generalized Bollobás-Erdős graphs, and formulate our corresponding results. We shall also establish some results concerning particular cases of this problem. In Section 4 we give the proofs.

In a continuation of this paper we shall investigate the problem where instead of $\alpha\left(G_{n}\right)=o(n)$ we assume the stronger condition that the maximum size of a $K_{p}$-free induced subgraph of $G_{n}$ is $o(n)$.

Since we will be able to state our main result only after having introduced some involved definitions, here we give a simplified version of it.

It is easy to see, that for every $L_{1}, \ldots, L_{r}$ there exists a minimum $\vartheta\left(L_{1}, \ldots, L_{r}\right)$ such that whenever $f(n)=o(n)$, then

$$
R T\left(n, L_{1}, \ldots, L_{r}, f(n)\right) \leq \vartheta\left(L_{1}, \ldots, L_{r}\right) n^{2}+o\left(n^{2}\right) .
$$

(The equality is attained for some functions $f(n)$ for which $f(n) / n \rightarrow 0$ sufficiently slowly. Of course, if $f(n) / n \rightarrow 0$ too fast, then this $\vartheta$ can be replaced by a smaller constant. Still, when we speak (sometimes a little loosely) about $R T\left(n, L_{1}, \ldots, L_{r}, o(n)\right)$, we will always mean the determination of this minimum $\vartheta$. 
Theorem 1. Given the integers $k_{1}, \ldots, k_{r} \geq 3$, for $R T\left(n, k_{1}, \ldots, k_{r}, o(n)\right)$ there exists a fixed $t$ and a sequence of asymptotically extremal graphs $\left(S_{n}\right)$ such that the vertices of $S_{n}$ can be partitioned into $t$ classes $X_{1}, \ldots, X_{t}$ where

$$
-e_{S_{n}}\left(X_{i}\right)=o\left(n^{2}\right) \text { for } i=1,2, \ldots, t,
$$

and for $1 \leq i<j \leq t$

- either $d_{S_{n}}\left(X_{i}, X_{j}\right)=\frac{1}{2}+o(1)$ or $d_{S_{n}}\left(X_{i}, X_{j}\right)=1+o(1)$.

To get some insight first we construct some graphs which later will turn out to be extremal in most of our problems.

The Erdös graph. The simplest statement in our field is as follows. If $\alpha\left(G_{n}\right)=o(n)$ and $K_{3} \nsubseteq G_{n}$, then $e\left(G_{n}\right)=o\left(n^{2}\right)$. One immediately wants to know if such graphs (with $\alpha\left(G_{n}\right)=o(n)$ and $K_{3} \nsubseteq G_{n}$ ) do exist at all. The existence of such graphs can be proved by using probabilistic arguments but there is a more useful way to get such graphs. In [14] Erdős and Rogers constructed a graph sequence $\left(G_{m}\right)$ for which $\alpha\left(G_{m}\right)=o(m)$ but $K_{3} \nsubseteq G_{m}$.

Combining the Erdős (or Erdọ̋-Rogers) graph with Turán's graph we get some very useful graph sequences.

Definition 2. (Canonical colouring with respect to a vertex-partition) Given a graph $G_{n}$ the vertex set of which is partitioned into the classes $X_{1}, \ldots, X_{q}$, an $r$-colouring will be called canonical, if the colour of an edge depends only on the classes its endpoints belong to: all the edges $(x, y): x, y \in X_{i}$ have the same colour $\chi_{\nu_{i}}$, and for $1 \leq i<j \leq q$ all the edges $(x, y): x \in X_{i}, y \in X_{j}$ have the same colour $\chi_{\nu_{i, j}}$.

Construction 1. Given the integer $d$, consider $T_{n, d}$, with the classes $C_{1}, \ldots, C_{d}$, and put into each $C_{i}$ an Erdös graph $H$ of $\left[\frac{n}{d}\right]$ vertices, with $\alpha(H)=o(n)$. Thus we get a graph $U_{n}=U(n, d)$ with $\alpha\left(U_{n}\right)=o(n)$.

(a) Since $K_{2 d+1} \nsubseteq U_{n}$, therefore

$$
R T(n, 2 d+1, o(n)) \geq e\left(T_{n, d}\right) .
$$

(b) Colouring the edges of $T_{n, d}$ by RED and the edges in the classes $C_{i}$ by BLUE we obtain a graph not containing RED $K_{d+1}$, nor BLUE $K_{3}$ :

$$
R T(n, d+1,3, o(n)) \geq e\left(T_{n, d}\right) .
$$

We shall prove (see Theorem 4) that this is asymptotically sharp for $d=2$ and $d=3$. However for $d=4$ we get the extremal sequence by the following:

Construction 2. Let $t=R(q, s)-1$ (where $R(q, s)$ is the Ramsey function). Colour $T_{n, t}$ by RED and BLUE canonically (with respect to the classes of $T_{n, t}$ ) so that the coloured graph should contain neither RED $K_{q}$, nor BLUE $K_{s}$. Put into each class of this graph a RED Erdös graph. Then the resulting graph $U_{n}=U(n, q, s)$ will contain neither a RED $K_{2 q-1}$, nor BLUE $K_{s}$. Clearly,

$$
e\left(U_{n}\right)>\left(1-\frac{1}{t}\right)\left(\begin{array}{l}
n \\
2
\end{array}\right)
$$

and 
Hence

$$
\alpha\left(U_{n}\right)=o(n)
$$

$$
R T(n, 2 q-1, s, o(n)) \geq e\left(T_{n, t}\right) .
$$

Let us list the already known upper bounds on $R T\left(n, k_{1}, \ldots, k_{r}, m\right)$. If $m=n$, then there is no real restriction on $\alpha\left(G_{n}\right)$. Put $T\left(n, k_{1}, \ldots, k_{r}\right):=$ $R T\left(n, k_{1}, \ldots, k_{r}, n\right)$. This case is described by

Theorem [22]. Let $k_{1}, \ldots, k_{r} \geq 3$ be given integers, and let $T\left(n, k_{1}, \ldots, k_{r}\right)$ denote the maximum number of edges an $r$-coloured $G_{n}$ can have under the condition that it does not contain a $K_{k_{\nu}}$ in its $\nu$ th colour. Then

$$
T\left(n, k_{1}, \ldots, k_{r}\right) \leq\left(1-\frac{1}{R\left(k_{1}, \ldots, k_{r}\right)-1}\right)\left(\begin{array}{l}
n \\
2
\end{array}\right) .
$$

This inequality immediately follows from Turán's and Ramsey's theorems. Indeed, if

$$
e\left(G_{n}\right)>\left(1-\frac{1}{R\left(k_{1}, \ldots, k_{r}\right)-1}\right)\left(\begin{array}{l}
n \\
2
\end{array}\right),
$$

then it contains a complete $K_{R}$ for $R=R\left(k_{1}, \ldots, k_{r}\right)$. This is coloured by $r$ colours, hence for some $\nu$ it contains a $K_{k_{\nu}}$ in the $\nu$ th colour.

The theorem is sharp up to an additive constant $O(1)$.

If $r=1$, then this theorem reduces to Turán's theorem.

Remark 1. Clearly, if $m>\frac{n}{R\left(k_{1}, \ldots, k_{r}\right)-1}$, then

$$
R T\left(n, k_{1}, \ldots, k_{r}, m\right)=T\left(n, k_{1}, \ldots, k_{r}\right) .
$$

On the other hand we shall always assume that $m$ is so large that $n<$ $R\left(k_{1}, \ldots, k_{r}, m\right)$.

The problem of $T\left(n, L_{1}, \ldots, L_{r}\right):=R T\left(n, L_{1}, \ldots, L_{r}, n\right)$ is still "easy":

Theorem. [7] Let $t=t\left(L_{1}, \ldots, L_{\tau}\right)$ be the smallest integer for which there exists a $v>0$ such that

$$
K_{t}(v, \ldots, v) \rightarrow\left(L_{1}, \ldots, L_{r}\right),
$$

(or, in less formal language), for any $r$-colouring of $K_{t}(v, \ldots, v)$ there exists a colour $\nu$ and a monochromatic $L_{\nu}$ in this colour. Then for some constant $c>0$,

$$
T\left(n, L_{1}, \ldots, L_{r}\right)=\left(1-\frac{1}{t-1}\right)\left(\begin{array}{l}
n \\
2
\end{array}\right)+O\left(n^{2-c}\right) .
$$

This is related to some results of Burr, Erdös and Lovász [7] and Chvátal. Applying their results and the Erdős-Stone Theorem [16] a slightly weaker form of the above theorem immediately follows. The proof of this version uses a strengthening of Erdős-Stone Theorem [16], the Erdös-Simonovits Theorem [8], [20], and is easy.

These are results where we used colourings but actually there were no restrictions on the independent sets. The first theorem where $\alpha\left(G_{n}\right)=o(n)$ was assumed is 
Theorem. [15] For $q=2 k+1$

$$
R T(n, q, o(n))=\left(1-\frac{1}{k}\right)\left(\begin{array}{l}
n \\
2
\end{array}\right)+o\left(n^{2}\right) .
$$

(See Construction 1.)

Intuitively the theorem asserts that for large $q$, if we add (in Turán's theorem on $K_{q}$ ) the extra condition that $\alpha\left(G_{n}\right)=o(n)$, this will have roughly the same effect as excluding a complete graph of half the original size.

For $q=3$ the theorem is trivial, since in that case $K_{3}$ is excluded, which implies that each degree is $o(n)$. The previous theorem leaves open the question of the even values, which is much more difficult. For quite a long while the question whether

$$
R T\left(n, K_{4}, o(n)\right)=o\left(n^{2}\right)
$$

seemed to be untractable. Then Szemerédi found a nontrivial upper bound:

Theorem. [23]

$$
R T\left(n, K_{4}, o(n)\right) \leq \frac{n^{2}}{8}+o\left(n^{2}\right)
$$

It came as a surprise - when Bollobás and Erdös proved - that (7) is sharp: Theorem. [2] (Construction)

$$
R T\left(n, K_{4}, o(n)\right) \geq \frac{n^{2}}{8}-o\left(n^{2}\right)
$$

Finally, the problem of $K_{2 k}$ and many related problems were settled in [10]. Theorem. [10] For $q=2 k$,

$$
R T\left(n, K_{q}, o(n)\right)=\frac{1}{2} \frac{3 q-10}{3 q-4} n^{2}+o\left(n^{2}\right) .
$$

\section{The Bollobás-Erdős graph and its generalization}

First we describe the original construction of Bollobás and Erdős used to prove (8). We shall fix $n$ points on the surface of a high dimensional unit sphere $\mathbf{S}$ "uniformly". Speaking of the relative measure of a set $X$ on $\mathbf{S}$ we will mean the measure of $X$ divided by the total measure of $\mathbf{S}$.

In the definition of the BE-graph [2] the following fact is used:

Given $\delta, \eta>0$, for any $\varepsilon>0$ small enough and integer $h>h_{0}(\varepsilon)$, if $\mathbf{S}$ is a unit sphere in the $h$-dimensional euclidean space $\mathbf{E}^{h}$, then for $\mu=\varepsilon / \sqrt{h}$

(*) for every sufficiently large $n \mathbf{S}$ can be partitioned into $n$ sets of equal measure and diameter $<\mu / 10$; 
(**) the relative measure of the spherical cap of diameter $2-\frac{1}{2} \mu$ is at most $\delta$;

$(* * *)$ given a point $x$, the relative measure of the set of points $y$ with $\rho(x, y)<$ $\sqrt{2}-\mu$ is almost $\frac{1}{2}$, more precisely, at least $\frac{1}{2}-\eta$.

Based on this, let us partition $\mathbf{S}$ into $\frac{n}{2}$ subsets $A_{1}, \ldots, A_{n / 2}$ as described in (*), select two sets of vertices, $X_{1}, X_{2}$ of size $\frac{n}{2}$, each containing exactly one point from each $A_{i}$, and then join two vertices $x$ and $y$ by an edge iff

(i) either $x \in X_{i}$ and $y \in X_{3-i}$, and $\rho(x, y)<\sqrt{2}-\mu$

(ii) or $x, y \in X_{i} i=1$ or 2 and $\rho(x, y) \geq 2-\mu$.

Denote the obtained graph by $B E(n, h, \varepsilon)$. It is not uniquely determined by the above construction, but any realization of it will have the properties we need:

(a) $\alpha(B E(n, h, \varepsilon))<2 \delta n$.

(b) the degrees of the vertices are $>\left(\frac{1}{2}-\eta\right) n$.

(c) $K_{4} \nsubseteq B E(n, h, \varepsilon)$.

(d) the subgraphs spanned by $X_{1}$ and $X_{2}$ are $K_{3}$-free.

In our main theorems the asymptotically extremal graph sequences will be given by some generalizations of the BE-graph. In this generalization we use many sets $X_{1}, \ldots, X_{t}$ on the sphere and of different sizes.

Definition 3. (Weighted $t$-partite $B E$ graphs, construction). The graph to be defined below will depend on the integers $h, t, n_{1}, \ldots, n_{t}$, and on a small positive number $\mu$, and will be denoted by

$$
B\left(h, t\left|n_{1}, \ldots, n_{t}\right| \mu\right) .
$$

We shall subdivide the points of the $h$-dimensional sphere $\mathbf{S}$ into $n_{i}$ sets according to $(*)$, for each $i$. For $i=1, \ldots, t, k=1, \ldots, n_{i}$ choose a vertex $x_{i k}$ from the $k$ th set of the $i$ th partition. Put $X_{i}=\left\{x_{i k}, 1 \leq k \leq n_{i}\right\}$ for $1 \leq i \leq t$ and let $V(B)=\bigcup_{i=1}^{t} X_{i}$. For each pair $x, y \in X_{i}$ we join them iff $\rho(x, y)>2-\mu$. For some pairs $(i, j)(i \neq j)$ we join every vertex of $X_{i}$ to every vertex of $X_{j}$, for all the other pairs we build a Bollobás-Erdós graph between the two classes: join $x \in X_{i}$ to $y \in X_{j}$ iff $\rho(x, y)<$ $\sqrt{2}-\mu$. The resulting graph is the monochromatic generalized Bollobás-Erdős or shortly GBE graph. If two classes are joined completely, we shall call this a "full" connection; if they are joined by a Bollobás-Erdös graph, we shall call this a "half" connection.

Remark 2. The graph $B\left(h, t\left|n_{1}, \ldots, n_{t}\right| \mu\right)$ is not completely determined by the parameters listed, even when the "full" connections and "half" connections are determined, since the embedding of the vertices into the sphere also can slightly influence its structure. However, in all our statements these minor variances can be neglected. We shall choose the parameters so that with $\varepsilon \rightarrow 0$ slowly enough and $h \rightarrow \infty$ (at a speed, depending on $\varepsilon$ ), $\mu=: \varepsilon / \sqrt{h}$ and $n>n_{0}(h, \varepsilon)$. Under these assumptions our assertions will not depend on the choice of these "hidden parameters". 


\section{Main results}

Our main results solve asymptotically the problem of $R T\left(n, k_{1}, \ldots, k_{r}, o(n)\right)$, (Theorem 2 ) and yield an upper bound on $R T\left(n, L_{1}, \ldots, L_{r}, o(n)\right)$, (Arboricity Theorem).

The structure of the asymptotically extremal graphs is given by

Theorem 2. Let $k_{1}, \ldots, k_{r} \geq 3$ be given integers, then for some fixed there exists a sequence of graphs $B\left(h, t\left|n_{1}, \ldots, n_{t}\right| \mu\right)$ asymptotically extremal in the problem of $R T\left(n, k_{1}, \ldots, k_{r}, o(n)\right)$. (Meanwhile $\mu \rightarrow 0$ and $h \rightarrow \infty$.)

Obviously Theorem 2 immediately implies Theorem 1.

Let $A$ be a $t \times t$ matrix, whose diagonal entries are all 0 's and $a_{i j}=\frac{1}{2}$ or 1 depending on whether the $i$ th and $j$ th classes of $B\left(h, t\left|n_{1}, \ldots, n_{t}\right| \mu\right)$ are joined by a $B E$-graph or completely. Let us normalize the integers $n_{1}, \ldots, n_{t}$ by putting

$$
u_{i}=\frac{n_{i}}{\sum n_{j}} \text {. }
$$

Then

$$
e\left(B\left(h, t\left|n_{1}, \ldots, n_{t}\right| \mu\right)\right)=u A u^{*} n^{2}+o\left(n^{2}\right)
$$

(as $h \rightarrow \infty, \varepsilon \rightarrow 0, n \rightarrow \infty$ ). If $u A u^{*}$ attains its maximum on the standard simplex

$$
\left\{\mathbf{u}: \quad \sum_{i=1}^{t} u_{i}=1, \quad u_{i} \geq 0(i=1, \ldots, t)\right\}
$$

on the boundary, i.e. for some $u_{k}=0$, then the $G B E$-graph will be called degenerate. If there are no maxima on the boundary, then one can easily see that there is exactly one maximum and the structure of this $G B E$-graph will be called dense.

An equivalent form of Theorem 2 is

Theorem 2'. Given the integers $k_{1}, \ldots, k_{r} \geq 3$, then for some fixed $t$ there exists a sequence of dense graphs $B\left(h, t\left|n_{1}, \ldots, n_{t}\right| \mu\right)$ asymptotically extremal for $R T\left(n, k_{1}, \ldots, k_{r}, o(n)\right)$.

Indeed, assume that Theorem 2 is already proved. If there exists a degenerate asymptotically extremal structure $B\left(h, t\left|n_{1}, \ldots, n_{t}\right| \mu\right)$ for the problem of $k_{1}, \ldots, k_{r}$, then we may assume that $n_{t}=0$, i.e. we got an asymptotically extremal structure with fewer classes. Consider an asymptotically extremal structure $B\left(h, t\left|n_{1}, \ldots, n_{t}\right| \mu\right)$ with minimum $t$. It must be dense.

Remark 3. Our problem is strongly related to multigraph and digraph extremal problems. The notion of dense structures was introduced by Brown, Erdös, and Simonovits in [3], (see also [4-6]).

To get some more information on $R T\left(n ; k_{1}, \ldots, k_{r}, o(n)\right)$ first we define the generalized complete graphs, then define a Ramsey number $\beta=\beta\left(k_{1}, \ldots, k_{r}\right)$ for generalized complete graphs, and finally prove that $\vartheta\left(k_{1}, \ldots, k_{r}\right)=\beta\left(k_{1}, \ldots, k_{r}\right)$.

Definition 4. (Generalized complete subgraphs) Let $R$ be a graph some vertices of which are "marked", the edges of which are weighted by the weights $0, \frac{1}{2}$ and 1 . 
$X \subseteq V(R)$ and $Y \subseteq X$ span (by definition) a generalized complete subgraph of the size $|X|+|Y|$ if

(a) all the vertices of $Y$ are "marked";

(b) all the edges in $Y$ have weight 1 ,

(c) all the edges in $X$ have weight $\geq \frac{1}{2}$.

Definition 5. (Weighted Ramsey numbers). Assume that the vertices and the edges of a $K_{t}$ are coloured by the colours $\chi_{1}, \ldots, \chi_{r}$, and the edges are weighted by $\frac{1}{2}$ and 1. For the colour $\chi_{\nu}$ we define $R^{\nu}$ as a weighted graph, where a vertex is "marked" iff its colour is $\chi_{\nu}$, and the edge $e$ gets weight $\frac{1}{2}$ or 1 if its colour is $\chi_{\nu}$ and its weight is $\frac{1}{2}$ or 1 respectively. (If its colour differs from $\chi_{\nu}$, then its weight is 0 in $R^{\nu}$.) Finally, assume that there is a distribution $\mathbf{u}=\left(u_{1}, \ldots, u_{t}\right)\left(u_{i}>0, \sum_{i=1}^{t} u_{i}=1\right)$ on the vertices of $K_{t}$. If we wish to emphasize that $K_{t}$ is weighted, we shall write $K_{t}(w)$. Let

$$
g\left(K_{t}(w), \mathbf{u}\right)=\sum_{1 \leq i<j \leq t} w_{i, j} u_{i} u_{j}
$$

where $w_{i, j}$ is the weight of $(i, j)$. We define the edge density of such a weighted graph as

$$
g\left(K_{t}(w)\right)=g\left(K_{t}(w), \mathbf{u}\right)=\max _{\mathbf{u}} \sum w_{i, j} u_{i} u_{j} .
$$

The weighted Ramsey number $\beta\left(k_{1}, \ldots, k_{r}\right)$ is the maximum $B$ such that

(*) there exist a $t$ and a weighted colouring of $K_{t}$ with edge density $B$ for which none of the subgraphs $R^{\nu}$ of colours $\chi_{\nu}$ (in $K_{t}$ ) contains a generalized $K_{k_{\nu}}$.

Clearly,

$$
t<R\left(k_{1}, \ldots, k_{r}\right) .
$$

Theorem 2". Let $k_{1}, \ldots, k_{r} \geq 3$ be given integers, then

$$
\vartheta\left(k_{1}, \ldots, k_{r}\right)=\beta\left(k_{1}, \ldots, k_{r}\right) \text {. }
$$

In other words,

$$
R T\left(n, k_{1}, \ldots, k_{r}, o(n)\right)=\beta\left(k_{1}, \ldots, k_{r}\right) n^{2}+o\left(n^{2}\right) .
$$

Corollary 1. There exists a finite algorithm to find an asymptotically extremal sequence for any $R T\left(n, k_{1}, \ldots, k_{r}, o(n)\right)$.

Motivation of the previous definitions. We shall consider a graph $G_{n}$ the edges of which are coloured with $r$ colours, and will approximate it by some (canonically coloured) $H_{n}=B\left(h, t\left|n_{1}, \ldots, n_{t}\right| \mu\right)$. This approximation will be "encoded" using a graph $R_{t}$ defined on the index set $\{1,2, \ldots, t\}$ of the classes $X_{i}$ of $H_{n}$, (see Definition 3). 
(a) The encoding goes as follows. If two sets $X_{i}$ and $X_{j}$ are joined completely, (in Definition 3 ) in RED, then $i$ and $j$ will be joined in $R_{t}$ by a RED edge of weight 1 . If they are joined by a RED $B E$-graph, $i$ and $j$ will be joined in $R_{t}$ by a RED edge of weight $\frac{1}{2}$. If the edges in $X_{i}$ are RED, "mark" $i$ in RED.

(b) This encoding will be useful, since the maximum size of a RED ordinary complete subgraph of $G_{n}$ will be at least as large as that of $B\left(h, t\left|n_{1}, \ldots, n_{t}\right| \mu\right)$ and this will be exactly as large as the maximum size of a RED generalized complete subgraph of $R_{t}$.

We shall characterize below the relative sizes of the classes $X_{i}, i=1, \ldots, t$ by the distribution vector $\mathbf{u}$.

(c) The size $|X|+|Y|$ of a generalized complete graph is used because if $R_{t}$ contains a generalized $K_{q}$ with the vertex-sets $X$ and $Y \subseteq X$ (as described above), then we shall be able to find a $K_{q} \subseteq G_{n}$ with $q=|X|+|Y|$ : each vertex of $Y$ will yield 2 vertices of this ordinary $K_{q}$ and each vertex of $X-Y$ will yield one vertex of $K_{q}$.

Until now we restricted our considerations to the case of complete graphs. As described in $[10], R T(n, L, o(n))$ depends in some sense on the arboricity of $L$ defined below - differently from the usual one - as follows. To consider arbitrary $L_{1}, \ldots, L_{r}$ in our problem, we need the

Definition of Arboricity. (a) $L \in A r b(2 k)$ if the vertices of $L$ can be $k$-coloured so that the subgraph spanned by the $\nu$ th colour is a forest, for $\nu=1,2, \ldots, k$.

(b) $L \in A r b(2 k+1)$ if the vertices of $L$ can be $k+1$-coloured so that the subgraph spanned by the $\nu$ th colour is a forest, for $\nu=1,2, \ldots, k$ and the vertices of colour $k+1$ are independent.

Remark 4. A slightly different definition of arboricity used to be given as the minimum $k$ for which $L$ can be coloured in $k$ colours so that each colour-class spans a forest.

Arboricity Theorem. Given $r$ graphs $L_{1}, \ldots, L_{r}$ with $L_{\nu} \in \operatorname{Arb}\left(k_{\nu}\right)$, then

$$
R T\left(n, L_{1}, \ldots, L_{r}, o(n)\right) \leq R T\left(n, k_{1}, \ldots, k_{r}, o(n)\right)+o\left(n^{2}\right) .
$$

Unfortunately, we have only upper bound for the general case. We do not even know the truth for $r=1$, not even for one of the simplest cases: we do not know if $R T(n, K(2,2,2), o(n))=o\left(n^{2}\right)$ or not. The Arboricity Theorem will not explicitly be proven here: we shall prove Theorem 2 and the reader can easily generalize the upper bound of Theorem 2 to the Arboricity Theorem, applying the Tree-building lemma, and the ideas given (in details) in [10].

We determine $R T\left(n ; L_{1}, L_{2}, o(n)\right)$ for some special $L_{1}, L_{2}$.

Definition 5. We shall say that for a problem $R T\left(n, L_{1}, \ldots, L_{k}, o(n)\right)$ the weak stability property holds if for any two sequences of asymptotically extremal graphs, say $\left(S_{n}\right)_{\infty}$ and $\left(Z_{n}\right)_{\infty}$ their distance is $o\left(n^{2}\right): \Delta\left(S_{n}, Z_{n}\right)=o\left(n^{2}\right)$. 


\section{Theorem 3.}

(a) $\vartheta\left(K_{3}, K_{3}\right)=\frac{1}{4} . \quad U(n, 2)$ is an asymptotically extremal sequence.

(b) $\vartheta\left(K_{3}, K_{4}\right)=\frac{1}{2}\left(1-\frac{1}{3}\right) . \quad U(n, 3)$ is an asymptotically extremal sequence.

(c) $\vartheta\left(K_{3}, K_{5}\right)=\frac{1}{2}\left(1-\frac{1}{5}\right) . \quad U(n, 3,3)$ is an asymptotically extremal sequence.

(d) $\vartheta\left(K_{4}, K_{4}\right)=\frac{1}{2}\left(1-\frac{3}{14}\right)$. The sequence in Construction 4 below is an asymptotically extremal structure.

(e) In all these cases the weak stability holds.

Theorem 4. If $p$ and $q$ are odd integers, then

$$
R T\left(n, C_{p}, C_{q}, o(n)\right)=\frac{n^{2}}{4}+o\left(n^{2}\right) .
$$

\section{Proof of Theorems 2-4}

Proof of the lower bound for $\vartheta\left(k_{1}, \ldots, k_{r}\right)$. Construction. There exists a correspondence between the coloured-weighted $K_{t}$ 's with distribution $\mathbf{u}$ and the graph sequences $\left\{B\left(h, t\left|n_{1}, \ldots, n_{t}\right| \mu\right)\right\}$.

Given a coloured weighted $K_{t}$ with a distribution (with a fixed $r$-colouring of the edges and vertices of $K_{t}$, with a distribution $u_{1}, \ldots, u_{t}$ on the vertices of $K_{t}$ and the weights $\frac{1}{2}$ and 1 on the edges), then there are canonically coloured generalized Bollobás-Erdős graphs $B\left(h, t\left|n_{1}, \ldots, n_{t}\right| \mu\right)$ corresponding to these data. Namely, given the dimension $h$ and $\mu>0$, fix the sets $X_{1}, \ldots, X_{t}$ on the $h$-dimensional sphere $\mathbf{S}$, so that

(a) $\left|X_{i}\right|=n_{i}=u_{i} n+o(n)$ and

(b) the set $X=X_{1} \cup \ldots \cup X_{t}$ be distributed as described in Definition 3 .

(c) take a "half" resp. a "full" connection between $X_{i}$ and $X_{j}$ if $w(i, j)=\frac{1}{2}$ resp. $w(i, j)=1$.

Obviously $e\left(B\left(h, t\left|n_{1}, \ldots, n_{t}\right| \mu\right)\right)=g\left(K_{t}(w), u\right) n^{2}+o\left(n^{2}\right)$.

We need also

Lemma 1. The above $B\left(h, t\left|n_{1}, \ldots, n_{t}\right| \mu\right)$ does not contain $K_{q}$ in colour $\chi_{\nu}$ iff the corresponding coloured weighted $K_{t}$ does not contain a generalized $K_{q}$ in colour $\chi_{\nu}$.

Proof. If $x_{1}, \ldots, x_{q}$ form a $\operatorname{RED} K_{q} \subseteq B\left(h, t\left|n_{1}, \ldots, n_{t}\right| \mu\right)$, then each $X_{i}$ contains at most 1 vertex of this $K_{q}$, unless $X_{i}$ is RED. Even so, it contains at most 2 vertices. Further, if $X_{i}$ and $X_{j}$ both contain 2 vertices of this $K_{q}$, then they must be RED and joined by RED edges completely. Hence the corresponding coloured, weighted $K_{t}$ contains a RED generalized $K_{q}$. This proves half of Lemma 1 . The other half is left to the reader. 
Now, given an arbitrary colouring $\phi$ of $K_{t}$ satisfying the property $(*)$ in Definition 5 (of $\beta\left(k_{1}, \ldots, k_{r}\right)$ ), build a $B\left(h, t\left|n_{1}, \ldots, n_{t}\right| \mu\right)$ with the corresponding colours and weight function $w$ and distribution $u$. Then - by Lemma 1 and the Definitions 3, and $5-K_{k_{\nu}} \nsubseteq B\left(h, t\left|n_{1}, \ldots, n_{t}\right| \mu\right)$ in colour $\chi_{\nu}$, and it will have

$$
\beta\left(k_{1}, \ldots, k_{r}\right) n^{2}+o\left(n^{2}\right)
$$

edges. (The error term comes from the fact that there is an error term in the BE-graph and that the vertices cannot be distributed exactly according to the distribution u.) Hence

$$
R T\left(n, k_{1}, \ldots, k_{r}, o(n)\right) \geq \beta\left(k_{1}, \ldots, k_{r}\right) n^{2}+o\left(n^{2}\right) .
$$

\section{Proof of the upper bound}

In the proof of the upper bound our first problem is that the condition $\alpha\left(G_{n}\right)=$ $o(n)$ applies only to the union of the colours, and so $G_{n}$ could contain $c n$ independent vertices in each separate colour. We need a lemma to overcome this difficulty.

Lemma 2. Fix an $\varepsilon>0$. If $G_{m}$ is an $r$-coloured graph with $\alpha\left(G_{m}\right)=\varepsilon m$, then one can partition the vertices of $G_{m}$ into $r+1$ classes $C_{0}, C_{1}, \ldots, C_{r}$ so that $\left|C_{0}\right|<2 \sqrt[r]{\varepsilon} m$ and for $1 \leq \nu \leq r$ for each $C_{\nu}$ every subset $Y \subset C_{\nu}$ of size $>\sqrt[r]{\varepsilon} m$ contains an edge of the $\nu$ th colour $\chi_{\nu}$.

Remark 5. One can ask if this partitioning is really necessary or under the condition of Lemma 2 there must always exist a colour $\chi$ and a constant $\eta$ tending to 0 as $\varepsilon \rightarrow$ 0 , for which every set of size $>\eta m$ contains an edge of colour $\chi$. The partitioning is needed. Let $G_{m}$ be the disjoint union of two Erdős graphs, one of which is coloured in RED, the other in BLUE. Then (fixing any $\eta \in\left(0, \frac{1}{2}\right)$ ), there is no fixed colour $\chi_{0}$ (=RED or BLUE) such that every vertex set $Y$ of size $>\eta m$ contains an edge of colour $\chi_{0}$.

Proof of Lemma 2. Let us assume that $G_{m}$ is coloured by $r$ colours: $\chi_{1}, \ldots, \chi_{r}$. For the sake of simplicity, we shall call $\chi_{1}$ RED, $\chi_{2}$ BLUE ... and $\chi_{r}$ BLACK. Let $\eta=$ $\sqrt[r]{\varepsilon}$ and call an $X \subseteq V\left(G_{m}\right)$ RED if

(a) $|X|>\varepsilon m$ and

(b) every $X^{*} \subseteq X$ of size $>\eta|X|$ contains a RED edge.

Clearly, the disjoint union of RED sets is RED. (However, a subset of a RED set is not necessarily RED). Fix a maximal RED subset of $V\left(G_{m}\right)$. Denote it by $V_{1}$. In the remaining part fix a maximal BLUE subset $V_{2}, \ldots$, and finally - in the $r$ th step - fix a maximal BLACK subset. Put $U_{0}=V\left(G_{m}\right)-\cup_{i} V_{i}$. If $\left|U_{0}\right| \leq \eta m$ then we are home.

Suppose that $\left|U_{0}\right|>\eta m$. If every $\eta m$-tuple of $U_{0}$ contains a RED edge, then we are home.

In the remaining case $U_{0}$ contains a $U_{1}$ of $>\eta m$ vertices but not containing RED edges. We shall use that - by the maximality of the $V_{i}$ no subsets of $U_{1}$ can be BLUE, ... or BLACK. Since $U_{1}$ is not BLUE, it contains a $U_{2}$ of $\eta^{2} m$ vertices but not containing BLUE edges. ... Since $U_{r-1}$ is not BLACK, it contains a $U_{r}$ of 
$>\eta^{r} m$ vertices but not containing BLACK edges. Clearly, $U_{r-1}$ is an independent set of $>\varepsilon m$ vertices, a contradiction.

Remark 6. It is easy to see that $\sqrt[r]{\varepsilon} m$ cannot be replaced by any essentially smaller value. To see this fix an integer $p$, put $q=p^{r}$ and $\varepsilon=\frac{1}{q}$. Take a Turán graph $T_{m, q}$, enumerate its classes by $r$-tuples $\left(p_{1}, \ldots, p_{r}\right)$, where $0 \leq p_{i}<p$. Colour the edges joining the class $C\left(a_{1}, \ldots, a_{r}\right)$ and $C\left(b_{1}, \ldots, b_{r}\right)$ with colour $j$ if the two indexsequences $a_{1}, \ldots, a_{r}$ and $b_{1}, \ldots, b_{r}$ differ first in the $j$ th position. In this graph each set of size $>\varepsilon m$ contains an edge, and one can easily see that with an $\eta<\frac{1}{(r+1) p}$ the lemma does not hold.

\section{Symmetrization}

One of the basic tools we shall use to prove our theorems is the symmetrization.

Let us modify Definition 5 by allowing (besides the weights $\frac{1}{2}$ and 1 ) the weight 0 as well. (This corresponds to allowing pairs of classes in $B\left(h, t\left|n_{1}, \ldots, n_{t}\right| \mu\right)$ not joined at all.) If there exists such a 0 weight in our graph, call the graph deficient. $\left(12^{*}\right)$, namely $t<R\left(k_{1}, \ldots, k_{r}\right)$ does not hold anymore for these deficient $K_{t}$ 's: the problem becomes infinite as soon as we allow deficient graphs. The next lemma tells us that for each deficient $K_{t}$ there exists a non-deficient one, at least as good as the deficient one.

For a (coloured) graph $G_{n}$ with the distribution $\mathbf{u}$ on the vertices and weights $\mathbf{w}$ on the edges let the weighted degree of a vertex $x$ be

$$
d(x)=n \sum_{(x, y) \in E\left(G_{n}\right)} w_{(x, y)} u_{y},
$$

where $E$ is the set of edges.

Lemma 3. Let $K_{t}, w$ and $\mathbf{u}$ be as in Definition 5, with the only exception that some weight, e.g., the weight of the edge $(x, y)$ be 0 . Let $d(x) \geq d(y)$. Then $g\left(\left(K_{t}-y\right)\left(w^{\prime}\right)\right) \geq g\left(K_{t}(w)\right)$ if $w^{\prime}$ is the weight on $K_{t}-y$ where the (original) weight of $y$ (in $K_{t}$ ) is added to the weight of $x$ (in $K_{t}-y$ ).

Proof. Trivial.

\section{Generalized Szemerédi Lemma.}

Szemerédi Lemma [24] asserts - loosely speaking - that given an $\varepsilon>0$, the vertex-set of every $G_{n}$ can be partitioned into a bounded number of classes so that almost all the pairs of classes will be $\varepsilon$-regular in the following sense.

Regularity condition. Given a graph $G_{n}$ and two disjoint vertex sets in it, $X$ and $Y$, we shall call the pair $(X, Y) \varepsilon$-regular if for every subset $X^{*} \subset X$ and $Y^{*} \subset Y$ satisfying $\left|X^{*}\right|>\varepsilon|X|$ and $\left|Y^{*}\right|>\varepsilon|Y|$,

$$
\left|d\left(X^{*}, Y^{*}\right)-d(X, Y)\right|<\varepsilon .
$$

The regularity condition means that the edges behave (in some weak sense) as if they were random. The following generalization asserts that if the edges of $G_{n}$ are coloured by a bounded number of colours, then one can find a partition for which the assertion of Szemerédi Lemma holds for each colour simultaneously. Below we formulate this lemma in the form we shall need it. Let $d_{\nu}(X, Y)$ denote the density in colour $\chi_{\nu}$. 
Generalized Regularity Lemma. For every $\varepsilon>0$, and integer $\lambda_{0}$ there exists a $\lambda_{\varepsilon}$ such that for every $r$-coloured $G_{n} V\left(G_{n}\right)$ can be partitioned into sets $V_{0}, V_{1}, \ldots, V_{\lambda}$ - for some $\lambda_{0}<\lambda<\lambda_{\varepsilon}$ - so that each $\left|V_{i}\right|<\varepsilon n,\left|V_{i}\right|=m$ (is the same) for every $i>$ 0 , and for all but at most $\varepsilon\left(\begin{array}{l}\lambda \\ 2\end{array}\right)$ pairs $(i, j)$, for every $X \subseteq V_{i}$ and $Y \subseteq V_{j}$, satisfying $|X|,|Y|>\varepsilon m$, we have

$$
\left|d_{\nu}(X, Y)-d_{\nu}\left(V_{i}, V_{j}\right)\right|<\varepsilon
$$

for every colour $\chi_{\nu}$.

This generalization does not seem to follow from the original lemma, however, the original proof can easily be modified to yield it. The role of $V_{0}$ is to make possible that all the other classes be exactly of the same size, and the role of $\lambda_{0}$ is to make the classes $V_{i}$ sufficiently small, so that - counting the edges - we could forget about the edges inside those classes.

Proof of the upper bound. (A) First we sketch the proof of the upper bound for $\vartheta\left(k_{1}, \ldots, k_{r}\right)$, (or, equivalently, for $\left.R T\left(n, k_{1}, \ldots, k_{r}, o(n)\right)\right)$, and also fix some parameters.

Let us fix an arbitrary $\varepsilon>0$. We shall also use a constant $\varepsilon_{1}>0$ which must be much smaller than $\varepsilon$, however, still fixed, and an $\varepsilon_{2}>0$ much smaller than $\varepsilon$ but much bigger then $\varepsilon_{1}$. If $S=k_{1}+\ldots+k_{r}$, then

$$
\varepsilon_{1}=(\varepsilon /(16 S))^{2 S}, \quad \varepsilon_{2}=(\varepsilon /(16 S))^{S}
$$

is an appropriate - perhaps too cautious - choice.

Assume that a graph sequence $\left(G_{n}\right)$ is given with $\alpha\left(G_{n}\right)=: \delta_{n} n=o(n)$. Let $\eta_{n}=\sqrt[r]{\delta_{n}}$. Here $\delta_{n}, \eta_{n} \rightarrow 0$. We shall fix an $n_{0}$ so that if $n>n_{0}$, then

$$
\eta_{n}<(\varepsilon / 16 S)^{4 S}
$$

Let $G_{n}$ be coloured by $r$ colours $\chi_{1}, \ldots, \chi_{r}$, and let $G_{n}^{\nu}$ be the graph spanned by the edges of colour $\chi_{\nu}$. We shall apply the Generalized Szemerédi Lemma to $G_{n}$, with $r, \varepsilon_{1}$ and a lower bound $\lambda_{0}=\frac{1}{\varepsilon}$ on the number of the classes.

(B) We shall prove that if $K_{k_{\nu}} \mathbb{E} G_{n}^{\nu},(\nu=1, \ldots, r)$ then

$$
e\left(G_{n}\right)<\beta\left(k_{1}, \ldots, k_{r}\right) n^{2}+3 r \varepsilon n^{2} .
$$

Below we sketch the proof and the details will be given in (C) and (D).

For each $G_{n}$ - for $n>n_{0}$, in paragraph $\left(\mathrm{C}_{5}\right)$ below - we shall define a weighted, coloured graph $H\left(w_{0}\right)$ (with a weight function $w_{0}$ and a distribution $\mathbf{u}_{0}$, and allowing also multiple edges) so that

$$
e\left(G_{n}\right) \leq g\left(H\left(w_{0}\right), \mathbf{u}_{0}\right) n^{2}+3 r \varepsilon n^{2}
$$

We shall call a weighted graph complete if any pair of vertices is joined by an edge of positive weight. $H\left(w_{0}\right)$ is not necessarily complete,

We shall apply symmetrization to $H\left(w_{0}\right)$ to obtain a weighted, coloured complete graph $K_{t}(w)$ satisfying

$\left(1^{*}\right) g\left(K_{t}(w), \mathbf{u}\right) \geq g\left(H\left(w_{0}\right), \mathbf{u}_{0}\right)$. 
$\left(2^{*}\right)$ If $K_{t}(w)$ contains a generalized complete $K_{q}$ in colour $\chi_{\nu}$, then $H\left(w_{0}\right)$ also contains a generalized complete $K_{q}$ in that colour and also an ordinary $K_{q} \subseteq$ $G_{n}^{\nu}$

$\left(3^{*}\right)$ Each pair of vertices of $K_{t}(w)$ is joined in exactly one of the colours $\chi_{1}, \ldots, \chi_{r}$ and with weight $\frac{1}{2}$ or 1 . Since $G_{n}^{\nu}$ contains no $K_{k_{\nu}}$, therefore $K_{t}(w)$ contains no generalized $K_{k_{\nu}}$ of colour $\chi_{\nu}$.

By $\left(3^{*}\right) K_{t}(w)$ occurs in the set of weighted coloured graphs in the definition of $\beta\left(k_{1}, \ldots, k_{r}\right)$. Hence $g\left(K_{t}(w)\right) \leq \beta\left(k_{1}, \ldots, k_{r}\right)$. By $\left(1^{*}\right)$, and $(18)$,

$$
e\left(G_{n}\right) \leq g\left(K_{t}(w)\right) n^{2}+2 r \varepsilon n^{2} \leq \beta\left(k_{1}, \ldots, k_{r}\right) n^{2}+3 r \varepsilon n^{2} .
$$

Obviously, this (together with the already obtained lower bounds (15)) will prove Theorem 2".

(C) Now we define $H\left(w_{0}\right)$.

$\left(\mathrm{C}_{1}\right)$ Using Lemma 2 for $n$ large enough we take a partition $V\left(G_{n}\right)=\bigcup_{\nu=0}^{r} U_{\nu}$ so that $\left|U_{0}\right|<\eta_{n} n$ and for $1 \leq \nu \leq r$ every $Y \subseteq U_{\nu}$, with $|Y|>\eta_{n} n$ contains an edge of colour $\chi_{\nu}$.

$\left(\mathrm{C}_{2}\right)$ Applying the Generalized Regularity Lemma to $G_{n}$, with the $\varepsilon_{1}>0$ and $\lambda_{0}$ (fixed above) we obtain the classes $V_{0}, V_{1}, \ldots, V_{T}$, with $\left|V_{i}\right|=m, 1 \leq i \leq T,\left|V_{0}\right|<$ $\varepsilon_{1} n$, (where $T \geq \lambda_{0}$ ). We delete $V_{0}$.

$\left(\mathrm{C}_{3}\right)$ Consider the "union" of the two partitions; the classes defined by $V_{i, j}:=$ $V_{i} \cap U_{j}, 1 \leq i \leq T, 1 \leq j \leq r$. Keep only the graph $G^{*}$ spanned by the vertex set

$$
V^{*}=\bigcup_{\left|V_{i, j}\right|>\varepsilon_{2} m} V_{i, j}
$$

We shall use the notation $W_{1}, \ldots, W_{M}$ for these classes $V_{i, j}$ in $V^{*}$.

Observe that we deleted at most $\left(\varepsilon_{2}+\eta_{n}\right) n$ points; $\left|V^{*}\right|>\left(1-\varepsilon_{2}-\eta_{n}\right) n$.

$\left(\mathrm{C}_{4}\right)$ For all but $r^{2} \varepsilon_{1}\left(\begin{array}{c}M \\ 2\end{array}\right)$ pairs $\left((i, j),\left(i^{\prime}, j^{\prime}\right)\right), i \neq i^{\prime}$, for every $\chi_{j}$

$$
\left|d_{\chi_{j}}(X, Y)-d_{\chi_{j}}\left(V_{i, \nu}, V_{i^{\prime}, j^{\prime}}\right)\right|<2 \varepsilon_{1}
$$

if $X \subseteq V_{i, j}, Y \subseteq V_{i^{\prime}, j^{\prime}},|X|,|Y|>\varepsilon_{1} m$. Indeed, if $\left(V_{i}, V_{i^{\prime}}\right)$ is a regular pair, then

$$
\begin{aligned}
\left|d_{\chi}(X, Y)-d_{\chi}\left(V_{i, j}, V_{i^{\prime} j^{\prime}}\right)\right| & \leq\left|d_{\chi}(X, Y)-d_{\chi}\left(V_{i}, V_{i^{\prime}}\right)\right| \\
& +\left|d_{\chi}\left(V_{i, j}, V_{i^{\prime}, j^{\prime}}\right)-d_{\chi}\left(V_{i}, V_{i^{\prime}}\right)\right|<2 \varepsilon_{1} .
\end{aligned}
$$

$\left(\mathrm{C}_{5}\right)$ Now we define a weighted, coloured graph $H\left(w_{0}\right)$ whose vertices are the classes $V_{i, j}, V_{i, j} \subset V^{*}$.

(i) We colour a vertex $W_{\ell}=V_{i, j}$ by colour $\chi_{\nu_{j}}$; (here $V_{i, j} \subset U_{j}$ ).

(ii) We assign the weight $u_{\ell}:=\mid \frac{W_{\ell} \mid}{V^{*} \mid}$ to the vertex $W_{\ell}, 1 \leq \ell \leq M$.

(iii) We join two classes $W_{\ell}=V_{i, j}, W_{\ell^{\prime}}=V_{i^{\prime}, j^{\prime}}$ in colour $\chi_{\nu}$ with weight 
$w_{\chi_{\nu}}\left(\ell, \ell^{\prime}\right)= \begin{cases}0, & \text { if } d_{\chi_{\nu}}\left(W_{\ell}, W_{\ell^{\prime}}\right)<2 \varepsilon \text { or }\left(V_{i}, V_{i^{\prime}}\right) \text { is not } \varepsilon_{1} \text {-regular or } i=i^{\prime} \\ \frac{1}{2}, & \text { if } 2 \varepsilon \leq d_{\chi_{\nu}}\left(W_{\ell}, W_{\ell^{\prime}}\right)<\frac{1}{2}+2 \varepsilon, i \neq i^{\prime} \text { and }\left(V_{i}, V_{i^{\prime}}\right) \text { is } \varepsilon_{1} \text {-regular } \\ 1, & \text { if } \frac{1}{2}+2 \varepsilon \leq d_{\chi_{\nu}}\left(W_{\ell}, W_{\ell^{\prime}}\right), i \neq i^{\prime} \text { and }\left(V_{i}, V_{i^{\prime}}\right) \text { is } \varepsilon_{1} \text {-regular }\end{cases}$

Below, comparing $e\left(G_{n}\right)$ and $g\left(H\left(w_{0}\right)\right) n^{2}$, we encounter 4 types of errors (typical in the applications of the Regularity Lemma):

- We have discarded a small number of vertices, $V-V^{*}$;

- We have discarded all the edges joining pairs in the same $V_{i}$ 's;

- We have replaced $e\left(V_{i}, V_{i^{\prime}}\right)$ by 0 for the irregular pairs;

- We had a "rounding" error $2 \varepsilon$ while counting the edges between a regular pair $\left(V_{i}, V_{i^{\prime}}\right)$.

There is still a fifth type of error, coming from the fact that we have many colours. Namely, we shall estimate $e\left(W_{\ell}, W_{\ell^{\prime}}\right)$ by

$$
\sum_{\ell, \ell^{\prime}} \sum_{\nu} w_{\chi_{\nu}}\left(\ell, \ell^{\prime}\right)\left|W_{\ell} \| W_{\ell^{\prime}}\right| \leq \sum_{\ell, \ell^{\prime}} \sum_{\nu} w_{\chi_{\nu}}\left(\ell, \ell^{\prime}\right) u_{\ell} u_{\ell^{\prime}} n^{2}
$$

where the "total density" $\sum_{\nu} w_{\chi_{\nu}}\left(\ell, \ell^{\prime}\right)>1$ can occur. In such cases we agree to replace some $w_{\chi_{\nu}}\left(\ell, \ell^{\prime}\right)$ 's by 0 to get $\sum_{\nu} w_{\chi_{\nu}}\left(\ell, \ell^{\prime}\right)=1$. Since $e\left(W_{\ell}, W_{\ell^{\prime}}\right) \leq\left|W_{\ell}\right|\left|W_{\ell^{\prime}}\right|$, the estimates below will still hold.

Clearly,

$$
e\left(G^{*}\right) \leq \sum_{\ell, \ell^{\prime}} \sum_{\nu} w_{\chi_{\nu}}\left(\ell, \ell^{\prime}\right) u_{\ell} u_{\ell^{\prime}} n^{2}+r \varepsilon n^{2}+r^{2} \varepsilon_{1} n^{2}+\frac{n^{2}}{T}
$$

where the term $r \varepsilon n^{2}$ comes from the "rounding" in the definition of $w_{\chi_{\nu}}\left(\ell, \ell^{\prime}\right)$, $r^{2} \varepsilon_{1} n^{2}$ estimates the number of edges corresponding to irregular pairs. The term $\frac{n^{2}}{T}<\varepsilon n^{2}$ represents the edges the endvertices of which belong to the same groups $V_{i}$.

From

$$
g\left(H\left(w_{0}\right), \mathbf{u}_{0}\right)=\sum_{\ell, \ell^{\prime}} \sum_{\nu} w_{\chi_{\nu}}\left(\ell, \ell^{\prime}\right) u_{\ell} u_{\ell^{\prime}}
$$

we have

$$
e\left(G^{*}\right) \leq g\left(H\left(w_{0}\right), \mathbf{u}_{0}\right) n^{2}+2 r \varepsilon n^{2}
$$

By $\left|V-V^{*}\right|<\left(\eta_{n}+(r+2) \varepsilon_{2}\right) n$,

$$
e\left(G_{n}\right)-e\left(G^{*}\right) \leq\left|V-V^{*}\right| n \leq\left(\eta_{n}+(r+2) \varepsilon_{2}\right) n^{2}<2 \varepsilon n^{2} .
$$

Therefore

$$
e\left(G_{n}\right) \leq g\left(H\left(w_{0}\right)\right) n^{2}+3 r \varepsilon n^{2}
$$

(D) Now we define a weighted, coloured $K_{t}$ which satisfies $\left(1^{*}\right),\left(2^{*}\right)$ and $\left(3^{*}\right)$.

$\left(\mathrm{D}_{1}\right)$ Assume that $W_{\ell}$ and $W_{\ell^{\prime}}$ are independent in $H(w)$, i.e., $w_{\chi_{\nu}}\left(W_{\ell}, W_{\ell^{\prime}}\right)=$ 0 for $1 \leq i \leq r$. Let $d\left(W_{\ell}\right) \geq d\left(W_{\ell^{\prime}}\right)$. Provide $W_{\ell}$ with the weight $u_{\ell}+u_{\ell^{\prime}}$ and delete $W_{\ell^{\prime}}$. Obviously this contraction will not decrease the edge-density and will 
not increase the maximum size of a generalized complete subgraph in any colour. (Actually, this is the symmetrization lemma.) We repeat this step until all the 0 densities disappear. And even then we repeat this deletion and weight shifting until we get a dense $K_{t}$.

$\left(D_{2}\right)$ We show that if for some $\ell, \ell^{\prime} w_{\chi_{\nu}}\left(\ell, \ell^{\prime}\right)>0$ for more than one $\nu$, then without increasing the maximum size of a generalized complete subgraph in any colour, we can replace all these coloured edges with one appropriately coloured edge of weight 1 .

Indeed, if $w_{\chi_{\nu}}\left(\ell, \ell^{\prime}\right)>0$ e.g. for $\nu, \nu^{\prime}$, corresponding to the RED and BLUE edges between $W_{\ell}$ and $W_{\ell^{\prime}}$, then we may assume that $W_{\ell}$ is not BLUE. We replace all the edges between $W_{\ell}$ and $W_{\ell^{\prime}}$ with a BLUE edge of multiplicity 1 . Obviously this cannot increase the maximum size of a generalized complete subgraph in any colour but BLUE. (As a matter of fact, there may be at most 2 such colours and both must have weight $\frac{1}{2}$.)

We show that it does not increase the maximum size of a $K_{q}$ in BLUE either.

Suppose that after this step the sets $X, Y$ define a BLUE generalized $K_{q}$. Then $W_{\ell}, W_{\ell^{\prime}} \in X$, and $W_{\ell} \notin Y$ because $W_{\ell}$ is not BLUE, (see Definition 4). Hence the contribution of $W_{\ell}$ to $|X|+|Y|$ is 1 , independently of the weight of ( $W_{\ell}, W_{\ell^{\prime}}$ ).

Clearly the edge-density will not increase at this step.

The only thing left to be proven is $\left(2^{*}\right)$ : if we choose the parameters as described in (A) and if a generalized RED $K_{q} \subseteq K_{t}(w)$, then (i) a generalized $\operatorname{RED} K_{q} \subseteq H\left(w_{0}\right)$, and (ii) (an ordinary) $K_{q} \subseteq G_{n}^{R E D}$. Here (i) is trivial.

Remember that the vertices of $H\left(w_{0}\right)$ represent classes of vertices of $G_{n}$ of size $>\varepsilon_{2} m$, and the pairs of positive weight $w_{0}$ have the regularity property on level $\varepsilon_{1}$. Let our generalized RED $K_{q}$ be spanned by the vertex sets $X$ and $Y \subset X$ (as in Definition 4.) Then, if $\varepsilon_{1}, \varepsilon_{2}$ and $\delta_{n}$ satisfy (16) and (17), then we can choose recursively 1 vertex from each class represented by the points in $X-Y$ and 2 vertices from each class represented by the points in $Y$ which form the RED $K_{q}$, as follows. Assume that we wish to choose the vertices e.g. from the classes $W_{1}, \ldots, W_{|X|}$ and have already chosen the corresponding vertices from $\ell$ classes $W_{1}, \ldots, W_{\ell}$ so that these vertices have at least $\varepsilon^{\ell}\left|W_{i}\right|$ common neighbours in $W_{i}, i=\ell+1, \ldots,|X|$. Denote the class of these common neighbours by $W_{i}^{\ell}$. Now we pick 1 or 2 vertices (according to the "plan") from $W_{\ell+1}$ so that they be joined to at least $\varepsilon^{\ell+1}\left|W_{i}\right|$ common neighbours in $W_{i}, i=\ell+2, \ldots,|X|$. This can be done because

- all the sets $W_{i}^{\ell}$ in this argument have $>\varepsilon_{1} m$ elements and are in some $V_{j}$. Thus the regularity lemma is applicable to them with $\varepsilon_{1}$.

- By the regularity, all but $S \cdot \varepsilon_{1} m$ vertices of $W_{\ell+1}$ are joined to each $W_{i}^{\ell}$ by their "typical" densities: $>2 \varepsilon$ when we wish to pick 1 vertex; by $>\frac{1}{2}+2 \varepsilon$ when we have to pick two vertices. Denote the set of these vertices by $W_{\ell+1}^{*}$. Now the first case is trivial, we may pick any of these vertices.

- In the second case $W_{\ell+1}^{*}$ is in $W_{R E D}$ and is too large to be independent in RED. Hence it contains a RED edge $u v$ such that at least $2 \varepsilon\left|W_{i}^{\ell+1}\right|$ vertices of $W_{i}^{\ell+1}$ are joined in $\operatorname{RED}$ to both $u$ and $v$. So we pick these $u$ and $v$ and go on with the recursion. 
Proof of Theorem 2'. It follows from the above argument and the construction in the proof of the lower bound.

Proof of Corollary 1, using Theorem 2". Basically we have to show that $\beta$ of Definition 5 can algorithmically obtained. Since the coloured $K_{t}$ we use to get $\vartheta\left(k_{1}, \ldots, k_{r}\right)$ contains no $K_{k_{\nu}}$ of colour $\chi_{\nu}$, therefore

$$
t<R\left(k_{1}, \ldots, k_{r}\right)
$$

(see $\left(12^{*}\right)$ ). Further, given a $t$, and the weights $w_{i j}$, we can find the optimum distribution

$$
\mathbf{u}=\left(u_{1}, \ldots, u_{t}\right) \quad\left(u_{i} \geq 0, \sum u_{i}=1\right)
$$

for which $g\left(K_{t}(w), u\right)$ is the maximum. This maximum was denoted by $g\left(K_{t}(w)\right)$ and called the density of $K_{t}(w)$. To find $\beta$ in the above definitions it is enough to regard finitely many values of $t$, finitely many colouring and weighting $(\chi, w)$ for each $t$, check if there is a "bad" colour (containing a $\left.K_{k_{\nu}}\right)$. If not, then " $g\left(K_{t}(w)\right)$ is good". Then we have to take the maximum of the good $g\left(K_{t}(w)\right)$ 's. Hence there is a finite algorithm to determine $\beta\left(k_{1}, \ldots, k_{r}\right)$.

Proof of Theorem 3. Theorem 3(a) is generalized to Theorem 4, therefore its proof will follow from that of Theorem 4 . The proof of the stability property in (a) - (d) is left to the reader.

Lemma 4. Let $B_{n}$ be a graph and $A$ a set of vertices in $B_{n}, m=n-|A|$. If $A$ contains $o\left(n^{2}\right)$ edges, and

$$
e\left(B_{n}-A\right) \leq\left(1-\frac{1}{j}\right)\left(\begin{array}{c}
m \\
2
\end{array}\right),
$$

then

$$
e\left(B_{n}\right) \leq\left(1-\frac{1}{j+1}\right)\left(\begin{array}{l}
n \\
2
\end{array}\right) .
$$

Or equivalently, if for a vertex $x \in K_{t}(w)$

$$
g\left(K_{t}(w)-x\right) \leq\left(1-\frac{1}{k-1}\right)
$$

then

$$
g\left(K_{t}(w)\right) \leq\left(1-\frac{1}{k}\right)
$$

The meaning of this lemma is that if we can delete a set of independent or almost independent vertices of a graph $G_{n}$ so that the resulting graph $G_{m}$ has only as many edges as the "best" $k$-1-chromatic graph on $m$ vertices, then $G_{n}$ has only as many edges as the "best" $k$-chromatic graph on $n$ vertices. The proof is trivial.

Lemma 5. [3] The optimal weight distribution of vertices of a dense $K_{t}(w)$ is attained when all the weighted degrees are equal. Then the density equals the half of this weighted degree.

Proof of Lemma 5. It follows easily using symmetrizations. See also [3]. 
Lemma 6. If in a dense $K_{t}(w)$ each vertex is incident to at least $\lambda$ half-edges, then

$$
2 g\left(K_{t}(w)\right) \leq\left(1-\frac{1}{t}-\frac{\lambda}{2 t}\right) .
$$

If there exist no $\lambda$-regular graph on $t$ vertices, then we have strict inequality:

$$
2 g\left(K_{t}(w)\right)<\left(1-\frac{1}{t}-\frac{\lambda}{2 t}\right)
$$

Proof of Lemma 6. Let $d(i), 1 \leq i \leq t$ be the weighted degree of the vertex $i$ in $K_{t}(w)$. Let $i_{1}, \ldots, i_{\lambda(i)}$ be the vertices joined to $i$ with a "half"-edge. For $1 \leq i \leq t$

$$
d(i)=\sum_{j \neq i} w(i, j) u_{j} \leq 1-u_{i}-\frac{1}{2}\left(u_{i_{1}}+\ldots+u_{i_{\lambda(i)}}\right)
$$

Since each $u_{j}$ occurs in at least $\lambda$ inequalities, summing the above inequalities for $1 \leq i \leq t$ we get

$$
\min _{1 \leq i \leq t} d(i) \leq \frac{1}{t} \sum_{i} d(i) \leq \frac{1}{t}\left(t-1-\frac{\lambda}{2}\right)=1-\frac{2+\lambda}{2 t}
$$

By Lemma 5 this proves Lemma 6 .

Below we formulate a lemma to calculate the density of some graph structures.

Lemma 7. Let - in $K_{t}(w)-q$ vertex-independent $K_{3}$ 's and $r$ independent edges be given, where the edges and triangles are also pairwise vertex independent. Assume that the edges in this system (i.e. the edges and the pairs in the triangles) have weight $\frac{1}{2}$. Then

$$
2 g\left(K_{t}(w)\right) \leq\left(1-\frac{6}{6 t-9 q-4 r}\right) .
$$

(There may be some further half-edges as well!)

Proof of Lemma 7. If we replace a half-edge by a full edge, that cannot decrease the density. Therefore we may assume that $3 q$ vertices are covered by $K_{3}$ 's; $2 r$ vertices are covered by the independent half-edges, and all the other edges are full edges. There are $s=t-3 q-2 r$ other vertices. This structure will be denoted by $W(q, r, s)$.

One could easily prove this lemma, using the Lagrange method. Below we shall use an equivalent but more combinatorial technique. One can easily prove that if we have two dense structures $A$ and $B$ and join each vertex of $A$ to each vertex of $B$ with weight 1 , then the obtained structure is dense again. Hence $W(q, r, s)$ is a dense structure.

Further, by Lemma 5, the optimum distribution u yielding $g\left(K_{t}(w)\right)$ can be characterized by the fact that all the weighted degrees are equal. If the optimum 
distribution is $x, x, \ldots, x, y, \ldots, y, z, \ldots, z$, the corresponding vertices of $K_{t}(w)$ satisfy the following equations:

$$
1-2 x=1-\frac{3}{2} y=1-z
$$

Hence, if $z=6 m$, then $x=3 m, y=4 m$. Thus $1=9 q m+8 r m+6(t-3 q-2 r) m=$ $(6 t-9 q-4 r) m$, and consequently,

$$
g\left(K_{t}(w)\right)=\frac{1}{2}\left(1-\frac{6}{6 t-9 q-4 r}\right) .
$$

In the proofs below we apply Theorem 2'. If $B_{n}=B\left(h, t\left|m, \ldots, n_{t}\right| \mu\right)$ is an asymptotically extremal sequence then we consider the corresponding coloured, weighted graph $K_{t}(w)$ defined in its proof.

We agree to call a $K_{s}$ RED (or BLUE) if its edges are RED (or BLUE) and this has nothing to do with the colours of the vertices.

Proof of Theorem 3(b). The lower bound is given by $U(n, 3)$ of Construction $1(\mathrm{~b})$. Let $B_{n}=B\left(h, t\left|n_{1}, \ldots, n_{t}\right| \mu\right)$ be an asymptotically extremal structure of $R T\left(n, K_{3}, K_{4}, o(n)\right)$, with its canonical extremal colouring. Let $K_{t}(w)$ be the corresponding weighted coloured complete graph.

Now, if in $K_{t}(w)$ there exists a BLUE point incident to a BLUE edge, then we have a BLUE $K_{3} \subset B_{n}$, and we are home. So we may assume that if there is a BLUE point $x_{1} \in K_{t}(w)$ at all, then all the edges joining it to the other vertices are RED. Let $X_{1}$ be the corresponding class of $B_{n}$. We may apply Lemma 4 to $B_{m}=B_{n}-X_{1}$ : it contains neither a BLUE $K_{3}$, nor a RED $K_{3}$. Therefore $e\left(B_{m}\right) \leq$ $\frac{1}{4} m^{2}+o\left(m^{2}\right)$, and consequently, $e\left(B_{n}\right) \leq \frac{1}{3} n^{2}+o\left(n^{2}\right)$.

Proof of Theorem $3(\mathbf{c})$. Let $\{1,2, \ldots, t\}$ be the vertices of $K_{t}(w)$.

If one of the vertices, say $x$ is BLUE, then all the edges $(x, y)$ of $K_{t}(w)$ are RED, otherwise we had a BLUE $K_{3}$ in $G_{n}$. Hence the remaining part contains neither a RED $K_{4}$, nor a BLUE $K_{3}$. By Theorem $4($ b), its density is at most $\frac{1}{2}\left(1-\frac{1}{3}\right)$, hence - by Lemma $4-g\left(K_{t}(w)\right)<\frac{1}{2}\left(1-\frac{1}{4}\right)<\frac{1}{2}\left(1-\frac{1}{5}\right)$.

So we may assume that all the vertices of $K_{t}(w)$ are RED. Hence

(*) $K_{t}(w)$ contains neither a BLUE $K_{3}$, nor a RED $K_{4}$ with edges of arbitrary weights. Hence $t \leq R(3,4)=9$.

(**) All the edges of a RED $K_{3}$ have weight $\frac{1}{2}$.

In case of $t \leq 5$ the density can be at most $\frac{1}{2}\left(1-\frac{1}{5}\right)$. This is achieved only when $t=5$ and all the edges are "full". Hence the colouring of $K_{5}$ is the colouring of $U(n, 3,3)$, the PENTAGONLIKE colouring: otherwise $(* *)$ would be violated.

Assume now that $t=6$. Since - by $(*)$ and Ramsey Theorem - there exists a RED $K_{3}$ in $K_{t}(w)$, therefore, by $(* *)$, its edges must be of weight $\frac{1}{2}$.

Apply Lemma 7 with $q=1, r=0$ :

$$
2 g\left(K_{t}\right) \leq 1-\frac{2}{2 t-3}<1-\frac{1}{5} \quad \text { if } \quad t=6 .
$$

Finally let $7 \leq t \leq \mathbf{9}$. 
1. Observe that each $x$ is joined to at most 3 other vertices in BLUE. Indeed, if $x$ is joined to $y_{1}, y_{2}, \ldots, y_{i}$, then - since the BLUE $K_{3}$ 's are excluded, $-y_{1}, \ldots, y_{i}$ form a complete RED graph. Thus $i<4$.

2. We show that for $t \geq 7$ each $x$ is incident to at least $t-5$ (RED) half edges. Indeed, if $x$ is joined to $y_{1}, \ldots, y_{i}$ in BLUE and to $z_{1}, \ldots, z_{j}$ in RED, and, say, $x z_{1}$ is a full RED edge, then it is not contained in RED triangles. Therefore $z_{1}$ is joined to all the other $z$ 's in BLUE. If there is another full RED edge, say $x y_{2}$, then $y_{2}$ is also joined to all the other $y$ 's in BLUE. Since there is no BLUE triangle, thus $j<3$ : there are at most 2 BLUE edges incident with $x$. Since there are at most 3 BLUE edges $x y$, - if 2 of these RED edges are full, then $t \leq 1+3+2=6$.

Since $t \geq 7, x$ is incident to at least $2 \mathrm{RED}$ edges (with weight $\frac{1}{2}$ ). So we can apply Lemma 6: $2 g\left(K_{t}(w)\right) \leq 1-\frac{2}{t}<1-\frac{1}{5}$ if $t \leq 9$.

Proof of Theorem 3(d). The following conditions must hold on the colouring of the extremal $B\left(h, t\left|n_{1}, \ldots, n_{t}\right| \mu\right)$.

(a) There are no 4 classes $C_{i}, C_{j}, C_{k}$ and $C_{m}$ joined in the same colour (i.e. forming a monochromatic $K_{4}$ in the reduced graph).

(b) If $\triangle=\left(C_{i}, C_{j}, C_{k}\right)$ is RED, then all its "vertices" $C_{i}, C_{j}$ and $C_{k}$ are BLUE (where a RED triangle means that the edges between the different groups are RED!)

(c) If $C_{i}$ and $C_{j}$ are RED, then either they form a RED Bollobás-Erdös graph or they are joined, by a BLUE edge.

(a')-(c') If we swap BLUE and RED, (a)-(c) still hold.

(As a matter of fact, the converse statement is also true:

if for $B\left(h, t\left|n_{1}, \ldots, n_{t}\right| \mu\right)$ (a-c') hold, then it contains no monochromatic $K_{4}$.)

(A) First we prove, that $t=6$.

1. Suppose that $t \leq 4$. Then

$$
2 g\left(K_{t}(w)\right) \leq\left(1-\frac{1}{4}\right)<\frac{11}{14} .
$$

2. Let $t=5$. If there is at least one "half" edge, then similarly as in the proof of $4(\mathrm{c})$ we get

$$
2 g\left(K_{t}(w)\right) \leq \frac{10}{13}<\frac{11}{14}
$$

Consequently, we may suppose, that all the edges are "full". In that case we shall prove that in any colouring of $K_{5}(w)$ - satisfying (a)--(c') - there must be a monochromatic $K_{4}$ in $B_{n}$.

The PENTAGONLIKE colouring is excluded, since in that case we can always find two classes of the same colour and a full connection between them in the same colour. This would yield a monochromatic $K_{4}$.

If the vertices $x_{1}, x_{2}, x_{3}$ of $K_{5}(w)$ form a RED triangle, then $x_{1}, x_{2}, x_{3}$ must be BLUE by (b).

By (a) there exists a BLUE "full" connection between $x_{4}$ and $x_{1}, x_{2}, x_{3}$. Hence (by (c)) $x_{4}$ must be RED. Similarly, $x_{5}$ is also RED. Hence (by (c')) they are joined by BLUE. 
There are at most two RED edges between $\left\{x_{1}, x_{2}, x_{3}\right\}$ and $\left\{x_{4}, x_{5}\right\}$. Otherwise say $x_{4}$ were joined to two of $x_{1}, x_{2}, x_{3}$ by RED edge and consequently, we would have a RED triangle, say $\left(x_{1}, x_{2}, x_{4}\right)$ with one RED vertex $x_{4}$, contradicting (b). There are at most three BLUE edges between $\left\{x_{1}, x_{2}, x_{3}\right\}$ and $\left\{x_{4}, x_{5}\right\}$ : otherwise say $x_{1}$ were joined to both $x_{4}$ and $x_{5}$ in BLUE, yielding a BLUE triangle $\left(x_{1}, x_{4}, x_{5}\right)$ incident to a BLUE vertex.

Since there are 6 edges between $\left\{x_{1}, x_{2}, x_{3}\right\}$ and $\left\{x_{4}, x_{5}\right\}, 5$ vertices with all "full-connections" cannot be properly coloured.

(B) Next we consider the case when $t>6$.

First we prove, that in an admissible colouring of $K_{t}(w)$ all the monochromatic triangles have the same colour. Indeed, assume that $x_{1}, x_{2}, x_{3}$ is a RED, $y_{1}, y_{2}, y_{3}$ is a BLUE triangle Then $x_{i}$ 's are BLUE and $y_{j}$ 's are RED vertices. At least 5 of the 9 connections $x_{i} y_{j}$ have the same colour, say RED. Thus we get a RED triangle $x_{i}, x_{k}, y_{j}$, contradicting (b).

Assume that there exists at least one BLUE vertex, say $x_{1}$, and all the triangles are also BLUE. Then there is no RED triangle. $x_{1}$ cannot be contained in a BLUE triangle, by (b'). This implies that $x_{1}$ has at most 2 BLUE connections: otherwise, say $x_{2}, x_{3}$ and $x_{4}$ were joined to $x_{1}$ in BLUE, and therefore $x_{2}, x_{3}, x_{4}$ were a RED triangle. Similarly, $x_{1}$ is joined to at most 3 other vertices in RED, otherwise we have a BLUE $K_{4}$ in $K_{t}(w)$. Hence $x_{1}$ can be joined to at most 5 other vertices, a contradiction.

So the only remaining case to be settled is when all the groups are RED, and all the triangles are BLUE.

First observe that in this case each vertex in $K_{t}(w)$ is joined to at least $t-6$ other vertices in RED: otherwise, say, $x_{1}$ were joined to the remaining 6 vertices in BLUE and 3 of these vertices would form a BLUE triangle, yielding with $x_{1}$ a BLUE $K_{4}$. Now (c) and Lemma 6 yields

$$
2 g^{\prime} \leq\left(1-\frac{1}{t}-\frac{t-6}{2 t}\right)=\frac{1}{2} \frac{t+4}{t} .
$$

Thus for $t>7$ we obtained that $2 g<11 / 14$, for $t=7$ we use the second part of the lemma: $t-6=1$ and there is no 1-regular graph on 7 vertices.

(C) Assume now that $t=6$.

If there exists exactly one half-connection, or two half-connections incident to the same vertex, say $x_{6}$, then we can apply $(\mathrm{A})$ to the remaining 5 vertices $x_{1}, \ldots, x_{5}$. We have seen that in that case one of the conditions (a-c') must be violated: there exists a monochromatic $K_{4}$.

So we have proved that there exist at least two independent "half- connections" among the 6 vertices.

To finish the proof we have to give a "good" colouring of such a weighted $K_{6}$ with 2 independent half edges and determine the optimal weight distribution, showing that its density is $\frac{1}{2} \cdot \frac{11}{14}$.

This is given by Construction 4 .

Construction 4. For $n=14 t+o(t)$ fix 6 groups $W_{1}, \ldots, W_{6}$ as follows:

$$
\left|W_{1}\right| \approx 2 t,\left|W_{2}\right| \approx 2 t,\left|W_{3}\right| \approx 3 t,\left|W_{4}\right| \approx 3 t,\left|W_{5}\right| \approx 2 t,\left|W_{6}\right| \approx 2 t .
$$


$W_{1}$ and $W_{2}$ form a RED Bollobás-Erdős graph, $W_{3}, W_{4}$, are also RED groups: we build RED Erdős graphs on them; however, they are joined completely, in BLUE. $W_{5}$, and $W_{6}$ form a BLUE Bollobás-Erdös graph. All the other pairs of classes are completely joined, as follows:

$$
\left(W_{1}, W_{3}\right),\left(W_{1}, W_{4}\right),\left(W_{2}, W_{3}\right),\left(W_{2}, W_{4}\right),\left(W_{1}, W_{5}\right),\left(W_{2}, W_{6}\right)
$$

are BLUE,

$$
\left(W_{3}, W_{5}\right),\left(W_{3}, W_{6}\right),\left(W_{4}, W_{5}\right),\left(W_{4}, W_{6}\right),\left(W_{1}, W_{6}\right),\left(W_{2}, W_{5}\right)
$$

are RED. One can easily check that there are two BLUE triangles but all their groups are RED and the shortest odd RED cycle is a pentagon, and each "full" RED edge has at least one BLUE endgroup, no "full" BLUE edge connects BLUE groups. Thus the resulting $B\left(h, 6\left|n_{1}, \ldots, n_{6}\right| \mu\right)$ contains no monochromatic $K_{4}$ in the above colouring and has only $o(n)$ independent vertices.

It is easy to check that in $(\mathrm{C})$ we have the optimal distribution and that $e(B)=$ $\frac{1}{2}\left(1-\frac{3}{14}\right) n^{2}+o\left(n^{2}\right)$.

Proof of Theorem 4. We shall call the "odd-girth" of $G$ the length of the shortest odd cycle in it. Put BLUE Erdős graphs of "odd-girth" $>p, q$ into the classes of a RED $T_{n, 2}$. The obtained $U_{n, 2}$ contains no short odd RED or BLUE cycles. This yields the (constructive) lower bound in Theorem 3.

Apply the proof methods of Theorem 2", (upper bound). If the reduced graph $H\left(w_{0}\right)$ contains a BLUE vertex and a BLUE edge incident to it, then $G_{n}$ contains a BLUE $C_{p}$, assumed that $\varepsilon=\varepsilon(p, q)$ is chosen appropriately. The same holds in RED. Hence in the reduced coloured graph $K_{t}(w)$ all the vertices have colours different from the colour of any edge. Therefore all the vertices are of the same colour, say RED, and all the edges are of the other colour, say BLUE. If $K_{t}$ contains a BLUE $K_{3}$, then $G_{n}$ contains a BLUE $K(p, p, p)$, a contradiction. Hence there are at most 2 classes: $t=2$.

\section{Open problems}

There are various intriguing open problems in connection with the above theorems. We list below some of them.

Problem 1. How large can $t=t(q, s)$ be if $B\left(h, t\left|n_{1}, \ldots, n_{t}\right| \mu\right)$ is asymptotically extremal for the problem $R T(n, q, s, o(n))$ ?

It is easy to see, that $t$ is at least exponentially large. Indeed, let $q=2 k+1, t=$ $R(k, s)$, and consider $U(n, t)$. Clearly, $U(n, t)$ contains neither RED $K_{q}$, nor BLUE $K_{s}$ and has $\left(1-\frac{1}{t}\right)\left(\begin{array}{l}n \\ 2\end{array}\right)$ edges. Further, all the $G B E$-graphs with fewer classes have fewer edges. Hence the extremal $G B E$-graph has $t \geq R\left(\left[\frac{q}{2}\right], s\right)$ classes, otherwise it has too few edges.

Problem 2. Determine $\vartheta\left(K_{q}, K_{3}\right)$.

Perhaps the following is true. 
Conjecture 1. $U(n, k, 3)$ of Construction 2 is extremal for $R T(n, 3, q, o(n))$ if $q=$ $2 k+1$.

One of the basic problems to be attacked is in general: Given a graph $L$, how the graph theoretic properties of $L$ influence $\vartheta(L)$ ? In case of ordinary graph extremal problems the chromatic number is the most important factor in determining ext $(n, L)$. The previous paper [10] showed that the behaviour of $\vartheta(L)$ largely depends on the arboricity of $L$. Still, it did not give a complete solution of the question. The case of $K_{3}(2,2,2)$ seemed to be the first real difficulty. Since $K_{3}(2,2,2) \in$ $\operatorname{Arb}(4)$, therefore

(see $[10])$.

$$
\vartheta\left(K_{3}(2,2,2)\right) \leq \vartheta\left(K_{4}\right)=\frac{1}{8}
$$

We cannot improve this bound.

Problem 3. Decide if $\vartheta\left(K_{3}(2,2,2)\right)=0$.

One way to settle this question would be to show that the Bollobás-Erdos graph (or some slight modification of it) contains no $K(2,2,2)$. We cannot decide even this (seemingly simple) question.

Perhaps replacing $o(n)$ by a slightly smaller functions, say by $f(n)=\frac{n}{\log n}$ one could get smaller upper bounds.

Problem 4. Is it true that for some $c>0$,

$$
R T\left(n, K_{4}, \frac{n}{\log n}\right)<\left(\frac{1}{8}-c\right) n^{2} ?
$$

Problem 5. Is it true that for $R T\left(n, k_{1}, \ldots, k_{r}, o(n)\right)$ the asymptotically extremal structure is weakly stable?

The same type of questions can be asked for hypergraphs. in $[17]$.

Some hypergraph results on Turán-Ramsey problem can be found in [15] and

\section{References}

[1] B. Bollobás: Extremal graph theory, Academic Press, London, 1978.

[2] B. BollobÁs and P. ERdös: On a Ramsey-Turán type problem, Journal of Combinatorial Theory, B 21 (1976), 166-168.

[3] W.G. Brown, P. ERdös and M. Simonovits: Extremal problems for directed graphs, Journal of Combinatorial Theory, 15B(1) (1973), 77-93.

[4] W. G. Brown, P. ERdős and M. Simonovits: Multigraph extremal problems, in: Problemes Combinatoires et Théorie des Graphes (ed. J. Bermond et al.), CNRS Paris, 1978.

[5] W. G. Brown, P. Erdös, and M. Simonovits: Inverse extremal digraph problems, Finite and Infinite Sets, Colloq. Math. Soc. J. Bolyai 37 Eger 1981, Akad. Kiadó, Budapest (1985), 119-156. 
[6] W. G. Brown, P. Erdös, and M. Simonovits: Algorithmic Solution of Extremal Digraph Problems, Trans. Amer. Math. Soc. 292 (1985), 421-449.

[7] S. BurR, P. Erdős and L. Lovísz: On graphs of Ramsey type, Ars Combinatoria, 1 (1976), 167-190.

[8] P. ERDős: On some new inequalities concerning extremal properties of graphs, Theory of Graphs, Proc. Coll. Tihany, Hungary (ed. P. Erdős and G. Katona), Acad. Press., N. Y. 1968, 77-81.

[9] P. ERdös: Remarks on a theorem of Ramsey, Bull. Res. Conne. Israel 7 (1957), 21-24.

[10] P. Erdös, A. Hajnal, Vera T. Sós, and E. Szemerédi: More results on RamseyTurán type problem Ramsey-Turán type problems, Combinatorica 3 (1983), $69-82$.

[11] P. Erdős, A. Meir, Vera T. Sós and P. Turán: On some applications of graph theory I, Discrete Math. 2 (1972), (3) 207-228.

[12] P. ERdós, A. MEIr, Vera T. Sós and P. Turán: On some applications of graph theory II. Studies in Pure Mathematics (presented to R. Rado) Academic Press, London, 1971, 89-99.

[13] P. Erdös, A. Meir, Vera T. Sós and P. Turán: On some applications of graph theory III, Canadian Math. Bulletin 15 (1972), 27-32.

[14] P. ERDös and C. A. Rogers: The construction of certain graphs, Canadian Journal of Math 1962.; or Art of Counting, MIT PRESS.

[15] P. ERdös and VERA T. Sós: Some remarks on Ramsey's and Turán's theorems, in: Combin. Theory and Appl. (P. Erdős et al eds) Colloq. Math. Soc. J. Bolyai 4 (1969), 395-404.

[16] P. ERdös and A. H. Stone: On the structure of linear graphs, Bull. Amer. Math. Soc. 52 (1946), 1089-1091.

[17] P. FrankL and V. RöDL: Some Ramsey-Turán type results for hypergraphs, Combinatorica 8 (1988), 323-332.

[18] Motzkin, E. G. Straus: Maxima for graphs and a new proof of a theorem of Turán, Canadian Journal of Math. 17 (1965), 533-540.

[19] F. P. Ramsey: On a problem of formal logic, Proc. London Math. Soc. 2nd Series, 30 (1930), 264-286.

[20] M. Simonovits: A method for solving extremal problems in graph theory, in: Theory of graphs, Proc. Coll. Tihany, (1966), (Ed. P. Erdős and G. Katona) Acad. Press, N.Y., 1968, 279-319.

[21] M. Simonovits: Extremal Graph Theory, in: Selected Topics in Graph Theory (eds Beineke and Wilson) Academic Press, London, New York, San Francisco, 161200. (1983).

[22] Vera T. Sós: On extremal problems in graph theory, in: Proc. Calgary International Conf. on Combinatorial Structures and their Application, (1969), 407-410.

[23] E. SzEMERÉDI: On graphs containing no complete subgraphs with 4 vertices (in Hungarian), Mat. Lapok 23 (1972), 111-116.

[24] E. SzemerÉdi: On regular partitions of graphs, in: Problemes Combinatoires et Théorie des Graphes (ed. J. Bermond et al.), CNRS Paris, 1978, 399-401.

[25] P. TURÁN: On an extremal problem in graph theory, Matematikai Lapok 48 (1941), 436-452 (in Hungarian), (see also [30]).

[26] P. Turán: On the theory of graphs, Colloq. Math. 3 (1954), 19-30, (see also [30]).

[27] P. TuRán: Applications of graph theory to geometry and potential theory, in: Proc. Calgary International Conf. on Combinatorial Structures and their Application, (1969), 423-434, (see also [30]).

[28] P. TuRÁn: Constructive theory of functions, in: Proc. Internat. Conference in Varna, Bulgaria, 1970 Izdat. Bolgar Akad. Nauk, Sofia, 1972, (see also [30]). 
[29] P. TURÁn: A general inequality of potential theory, Proc, Naval Research Laboratory, Washington, (1970), 137-141, (see also [30]).

[30] Collected works of Paul Turán, Akadémiai Kiadó, Budapest, 1989.

[31] A. A. ZYKov: On some properties of linear complexes, Mat Sbornik, 24 (1949), 163-188; Amer. Math. Soc. Translations 79, 1952.

P. Erdős, A. Hajnal

M. Simonovits, V. T. Sós,

E. Szemerédi

Mathematical Institute of the

Hungarian Academy of Sciences,

Budapest

h1465haj@ella.hu

h1143simeella.hu

h1014soseella.hu

szemeredearamis. rutgers . edu 